\title{
AIAA 2001-0168
}

Response Surface Modeling Using Multivariate Orthogonal Functions

E.A. Morelli and R. DeLoach

NASA Langley Research Center

Hampton, VA

39th AIAA Aerospace Sciences Meeting \& Exhibit January 8-11, 2001

Reno, Nevada 


\title{
RESPONSE SURFACE MODELING USING MULTIVARIATE ORTHOGONAL FUNCTIONS
}

\author{
Eugene A. Morelli ${ }^{*}$ and Richard DeLoach ${ }^{\dagger}$ \\ NASA Langley Research Center \\ Hampton, Virginia USA 2368I-2199
}

\begin{abstract}
A nonlinear modeling technique was used to characterize response surfaces for non-dimensional longitudinal aerodynamic force and moment coefficients, based on wind tunnel data from a commercial jet transport model. Data were collected using two experimental procedures - one based on modern design of experiments (MDOE), and one using a classical one factor at a time (OFAT) approach. The nonlinear modeling technique used multivariate orthogonal functions generated from the independent variable data as modeling functions in a least squares context to characterize the response surfaces. Model terms were selected automatically using a prediction error metric. Prediction error bounds computed from the modeling data alone were found to be a good measure of actual prediction error for prediction points within the inference space. Root-mean-square model fit error and prediction error were less than 4 percent of the mean response value in all cases. Efficacy and prediction performance of the response surface models identified from both MDOE and OFAT experiments were investigated.
\end{abstract}

\footnotetext{
${ }^{*}$ Research Enginecr, Senior Member
}

$\dagger$ Senior Research Scientist

Copyright $\bigcirc 2001$ by the American Institute of Aeronautics and Astronautics, Inc. No copyright is asserted in the United States under Title 17, U.S. Code. The U.S. Government has a royaltyfree license to exercise all rights under the copyright claimed herein for Governmental purposes. All other rights are reserved by the copyright owner

\section{Nomenclature}

a

$E\{\}$

Cov

$J$

$M$

MDOE

n

$N$

OFAT

PSE

$p$

$w$

$x$

$y$

$\alpha$

$\gamma$

$\sigma^{2}$

8

superscripts

T

ง

$-1$

$\sim$

subscripts

$\begin{array}{ll}\max & \text { maximum } \\ \min & \text { minimum } \\ 0 & \end{array}$

variance

transpose

estimate

normalized

nominal parameter vector expectation operator

covariance matrix

cost function

Mach number

Modern Design Of Experiments

number of model terms

total number of samples

One Factor At a Time

predicted squared error

modeling function vector

ordinary polynomial function vector

independent variable vector

measured output vector

angle of attack, deg

orthogonalization scalar

vector element-by-element product

matrix inverse 


\section{Introduction}

A fundamental scientific activity is to attempt to find a mathematical description for the dependence of an output variable on independent variables that are varied during an experiment. For static tests, this mathematical description or model can be thought of geometrically as a hyper-surface, also called a response surface. Critical issues for successfully identifying an adequate response surface model from experimental data include the experiment design (or, how the independent variable values are set when measuring the output variable response), noise level on the measured output, identification of a mathematical model structure that can capture the functional dependence of the output variable on the independent variables, accurate estimates of unknown parameters in the identified model structure, and the ability of the identified model to predict output values for data that were not used to identify the response surface model.

An important application area for experiment design and response surface modeling is wind tunnel testing. Since 1997, wind tunnel testing technology activities conducted and sponsored by NASA Langley Research Center have included applying formal experiment design principles to empirical aerodynamics ${ }^{1}$. These activities bring to bear on aerodynamic research problems the powerful machinery of formal experiment design first introduced by R.A. Fisher and associates early in the $20^{\text {th }}$ century ${ }^{2}$ and used successfully since then in a wide range of industrial, scientific, and engineering applications. Collectively, these methods are described at NASA Langley as Modern Design of Experiments (MDOE), after a phrase from the literature of formal experiment design that distinguishes these methods from what is commonly called classical experiment design.

Classical experimentation methods have been popular for hundreds of years, and form the basis of conventional wind tunnel testing procedures in use today. The defining feature of classical testing methods is an error control strategy that requires each independent variable to be changed one at a time, while holding all other variables constant. This method, formally described in the literature of experiment design as One Factor At a Time (OFAT) testing, typically involves changing the levels of the independent variable under study as a monotonically increasing function of time. This is the basis of the common polar, for example, which is a popular wind tunnel testing data structure that consists of a series of angle of attack levels set sequentially in a monotonically increasing sequence, with all other independent variables (Mach number, angle of sideslip, etc.) held constant. The OFAT experiment design has been used extensively for wind tunnel tests, so that many wind tunnel facilities have computerized data collection systems designed to efficiently conduct experiments designed in this way.

The focus of response surface modeling is on defining a relationship between the output variable (also called a response variable or a dependent variable) and the independent variables (also called factors) that are changed during the experiment. If this relationship is characterized well, it is possible to predict responses for any combination of independent variables in the range of those tested, not simply the specific points set in the test. Furthermore, such mathematical relationships, called response surface models, can be used to predict responses in other circumstances, e.g., in other tests, or in flight. The MDOE approach to experiment design adopts tactics intended to make errors independent and identically distributed. In this way, the effects of local singlepoint errors resulting from the combined effects of all inevitable failures to precisely implement desired experimental conditions, can be made to substantially cancel. This results in higher precision than can be achieved for single points. Furthermore, the MDOE approach reduces the number of data points required to define the response surface, so the excess data points are available to assess the quality of predictions made by the model.

Typically, once the experimental data are collected, polynomials in the independent variables are used to model the functional dependence of the output variable on the independent variables, and the model parameters are estimated from the measured data using least squares linear regression ${ }^{3.4}$. Unfortunately, the question of which polynomial terms should be included in the model for a given set of data is often addressed by trial-and-error, or by just including all polynomial terms that could possibly be identified from the data, based on information limitations. A simple example of an information limitation is the general statement that a quadratic polynomial will fit 3 data points exactly, but a cubic polynomial, with its 4 model parameters, cannot be identified from just 3 data points. This problem of which modeling functions to include in the linear regression model, called model structure determination, gets more difficult as the range of the independent variables for the model increases or the complexity of the underlying functional dependency increases. 
Various stepwise regression techniques ${ }^{4.5}$ can be used to identify an adequate model structure, but these methods are iterative and require the involvement of an experienced analyst. Neural networks using radial basis functions with subspace partitioning, or back propagation with layered and interconnected nonlinear activation functions, have also been applied to the response surface modeling problem ${ }^{6}$. For this type of approach, there is a loss of physical insight and a danger of overfitting the data, because the model structures used contain many parameters, typically with no mechanism for limiting the size of the model other than the judgment of the analyst.

In this work, a nonlinear multivariate orthogonal modeling technique ${ }^{7}$ was used to model response surfaces for wind tunnel data. The technique generates nonlinear orthogonal modeling functions from the independent variable data, and uses those modeling functions with a predicted squared error metric to determine appropriate model structure. The orthogonal functions are generated in a manner that allows them to be decomposed without ambiguity into an expansion of ordinary multivariate polynomials. This allows the identified orthogonal function model to be converted to a multivariate ordinary polynomial expansion in the independent variables, which provides physical insight into the identified functional dependencies.

The next section gives the problem statement and describes the necessary theory. Following this, the multivariate orthogonal function modeling method is applied to identify response surface models for non-dimensional longitudinal aerodynamic force and moment coefficients, based on wind tunnel data from a commercial jet transport model.

\section{Theoretical Development}

The multivariate orthogonal function modeling approach presented here was first developed in Ref. [7].

Assume an $\mathrm{N}$-dimensional vector of dependent variable values, $\boldsymbol{y}=\left[y_{1}, y_{2}, \ldots, y_{N}\right]^{T}$, modeled in terms of a linear combination of $n$ modeling functions $\boldsymbol{p}_{j}, j=1,2, \ldots, n$. Each $\boldsymbol{p}_{j}$ is an $N$-dimensional vector which in general depends on the independent variables. Then,

$$
\boldsymbol{y}=a_{1} \boldsymbol{p}_{1}+a_{2} \boldsymbol{p}_{2}+\ldots+a_{n} \boldsymbol{p}_{n}+\varepsilon
$$

The $a_{j}, j=1,2, \ldots, n$ are constant model parameters to be determined, and $\varepsilon$ denotes the modeling error vector. Eq. (1) represents the usual mathematical model used to fit a response surface to measured data from an experiment. We put aside for the moment the important questions of determining how candidate modeling functions $\boldsymbol{p}_{j}$ should be computed from the independent variables, as well as which candidate modeling functions should be included in Eq. (1), which implicitly determines $n$. Now define an $N x n$ matrix $\boldsymbol{P}$,

$$
\boldsymbol{P}=\left[p_{1}, p_{2}, \ldots, p_{n}\right]
$$

and let $\boldsymbol{a}=\left[a_{1}, a_{2}, \ldots, a_{n}\right]^{T}$. Eq. (1) can be written as a standard least squares regression problem,

$$
y=\boldsymbol{P} a+\varepsilon
$$

where $y$ is a vector of measured dependent variable values and $\boldsymbol{P}$ is a matrix whose columns contain modeling functions of the measured independent variables, and $a$ is a vector of unknown parameters. The variable $\varepsilon$ represents a vector of errors that are to be minimized in a least squares sense. The least squares cost function is

$$
J=(\boldsymbol{y}-\boldsymbol{P} \boldsymbol{a})^{T}(\boldsymbol{y}-\boldsymbol{P a})=\varepsilon^{T} \boldsymbol{\varepsilon}
$$

The parameter vector estimate that minimizes this cost function is computed from ${ }^{1-4}$

$$
\hat{\boldsymbol{a}}=\left[\boldsymbol{P}^{T} \boldsymbol{P}\right]^{-1} \boldsymbol{P}^{T} \boldsymbol{y}
$$

The estimated parameter covariance matrix is

$$
\operatorname{Cov}(\hat{a})=E\left\{(\hat{\boldsymbol{a}}-\boldsymbol{a})(\hat{\boldsymbol{a}}-\boldsymbol{a})^{T}\right\}=\sigma^{2}\left[\boldsymbol{P}^{T} \boldsymbol{P}\right]^{-1}
$$

where $E$ is the expectation operator, and the equation error variance $\sigma^{2}$ can be estimated from the residuals,

$$
v=\boldsymbol{y}-\boldsymbol{P} \hat{a}
$$

$$
\hat{\sigma}^{2}=\frac{1}{(N-n)}\left[(\boldsymbol{y}-\boldsymbol{P} \hat{\boldsymbol{a}})^{T}(\boldsymbol{y}-\boldsymbol{P} \hat{\boldsymbol{a}})\right]=\frac{\boldsymbol{v}^{T} \boldsymbol{v}}{(N-n)}
$$


and $n$ is the number of elements in parameter vector $\boldsymbol{a}$. Parameter standard errors are computed as the square root of the diagonal elements of the $\operatorname{Cov}(\hat{a})$ matrix from Eq. (6), using $\hat{\sigma}^{2}$ from Eq. (8).

Estimated model output is

$$
\hat{\boldsymbol{y}}=\boldsymbol{P} \hat{\boldsymbol{a}}
$$

For response surface modeling, the modeling functions (columns of $\boldsymbol{P}$ ) are often chosen as polynomials in the measured independent variables. This approach corresponds to using the terms of a Taylor series expansion to approximate the functional dependence of the output response variable on the independent variables.

If the modeling functions are instead multivariate orthogonal functions generated from the measured independent variable data, advantages accrue in the model structure determination for response surface modeling. After the model structure is determined using the multivariate orthogonal modeling functions, each retained modeling function can be decomposed into an expansion of ordinary polynomials in the independent variables. Combining like terms from this final step puts the final model in the standard form of a Taylor series expansion. It is this latter form of the model that provides the physical insight, particularly in the case of modeling non-dimensional aerodynamic force and moment coefficients. This is the reason that aircraft dynamics and control analyses are nearly always conducted with the assumption of this form for the dependence of the non-dimensional aerodynamic force and moment coefficients on independent variables such as angle of attack and Mach number.

\section{Multivariate Orthogonal Function Modeling}

Orthogonal modeling functions $\boldsymbol{p}_{j}$ have the following important property:

$$
p_{i}^{T} p_{j}=0, i \neq j \quad, \quad i, j=1,2, \ldots, n
$$

Using Eqs. (2) and (10) in Eq. (5), the $j^{\text {th }}$ element of the estimated parameter vector $\hat{a}$ is given by

$$
\hat{a}_{j}=\left(\boldsymbol{p}_{j}^{T} \boldsymbol{y}\right) /\left(\boldsymbol{p}_{j}^{T} \boldsymbol{p}_{j}\right)
$$

Combining Eqs. (2), (4), and (9)-(10),

$$
\hat{J}=\boldsymbol{y}^{T} \boldsymbol{y}-\sum_{j=1}^{n} \hat{a}_{j}^{2}\left(\boldsymbol{p}_{j}^{T} \boldsymbol{p}_{j}\right)
$$

or, using Eq. (11),

$$
\hat{J}=y^{T} y-\sum_{j=1}^{n}\left(p_{j}^{T} y\right)^{2} /\left(p_{j}^{T} p_{j}\right)
$$

Eq. (13) shows that when the modeling functions are orthogonal, the reduction in the estimated cost resulting from including the term $a_{j} \boldsymbol{p}_{j}$ in the model depends only the dependent variable data $y$ and the added orthogonal modeling function $\boldsymbol{p}_{j}$. This decouples the least squares modeling problem, and makes it possible to evaluate each orthogonal modeling function in terms of its ability to reduce the least squares model fit to the data, regardless of which other orthogonal modeling functions are present in the model. When the modeling functions $\boldsymbol{p}_{j}$ are instead polynomials in the independent variables (or any other non-orthogonal function set), the least squares problem is not decoupled, and iterative analysis is required to find the subset of modeling functions necessary for an adequate model structure.

The orthogonal modeling functions to be included in the model are chosen to minimize predicted squared error PSE, defined by 8

$$
\mathrm{PSE}=\frac{(\boldsymbol{y}-\boldsymbol{P} \hat{\boldsymbol{a}})^{T}(\boldsymbol{y}-\boldsymbol{P} \hat{a})}{N}+2 \sigma_{\max }^{2} \frac{n}{N}
$$

or

$$
\operatorname{PSE}=\frac{\hat{J}}{N}+2 \sigma_{\max }^{2} \frac{n}{N}
$$

where $\sigma_{\max }^{2}$ is the maximum variance of elements in the error vector $\varepsilon$, assuming the correct model structure. The PSE in Eq. (15) depends on the mean squared fit error $\hat{J} / N$, and a term proportional to the number of terms in the model, $n$. The latter term is a model overfit penalty that prevents overfitting the data with too many model terms, which is detrimental to model prediction accuracy ${ }^{8}$. The factor of 2 in the model overfit penalty term accounts for the fact that the PSE is being used when the model structure is not correct, i.e., during the model structure determination 
stage. Ref. [8] contains further justifying statistical arguments and analysis for the form of PSE in Eqs. (14)-(15). Note that while the mean squared fit error $\hat{J} / N$ must decrease with the addition of each orthogonal modeling function to the model (by $\mathrm{Eq}$. (12) or (13)), the overfit penalty term $\sigma_{\max }^{2} n / N$ increases with each added model term ( $n$ increases). Introducing the orthogonal modeling functions into the model in order of most effective to least effective in reducing the mean squared fit error (quantified by $\hat{a}_{j}^{2}\left(\boldsymbol{p}_{j}^{T} \boldsymbol{p}_{j}\right)$ for the $j^{\text {th }}$ orthogonal modeling function) means that the PSE metric will always have a single global minimum value. Figure 1 depicts this graphically, using actual modeling results from one of the cases discussed later. Ref. [8] contains details on the statistical properties of the PSE metric, including justification for its use in modeling problems.

For wind tunnel testing, repeated runs at the same test conditions are often available. If $\sigma_{o}^{2}$ is the output variance estimated from measurements of the output for repeated runs at the same test conditions, then $\sigma_{\max }^{2}$ can be estimated as

$$
\sigma_{\max }^{2}=25 \sigma_{o}^{2}
$$

If the output errors were Gaussian, Eq. (16) would correspond to conservatively placing the maximum output variance at 25 times the estimated value (corresponding to a $5 \sigma_{o}$ maximum deviation). However, the output errors are in general not Gaussian, because PSE is used to evaluate candidate model structures. In addition, the estimate of $\sigma_{o}^{2}$ may not be very good, because of relatively few repeated runs available and inevitable errors in duplicating test conditions. These are the reasons for choosing $5 \sigma_{o}$ for the maximum deviation in Eq. (16). The model structure determined using PSE was found to be virtually the same for $\sigma_{\max }^{2}$ in the range:

$$
9 \sigma_{o}^{2} \leq \sigma_{\max }^{2} \leq 100 \sigma_{o}^{2}
$$

Using orthogonal functions to model the dependent variable made it possible to evaluate the merit of including each modeling function individually as part of the model, using the predicted squared error PSE. Since the goal is to select a model structure with minimum PSE, and the PSE always has a single global minimum for orthogonal modeling functions, the model structure determination was a well-defined and straightforward process that could be (and was) automated.

After the orthogonal modeling functions that minimized PSE were selected, each retained orthogonal function was expanded into an ordinary polynomial expression, and common terms in the ordinary polynomials were combined using double precision arithmetic to arrive finally at a multivariate model using only ordinary polynomials in the independent variables. This procedure is explained below in the Parameter Estimation section.

Orthogonal modeling functions are useful in determining the model structure for the dependent variable using the PSE metric, by virtue of the properties of orthogonal modeling functions and the resultant decoupling of the associated least squares problem. The subsequent decomposition of the retained orthogonal functions is done to express the results in physically meaningful terms and to allow analytic differentiation for partial derivatives of the dependent variable with respect to the independent variables.

The next section describes a procedure for using the independent variable data to generate orthogonal modeling functions, which have the orthogonality property of Eq. (10).

\section{Orthogonal Function Generation}

The technique for generating orthogonal functions of several independent variables based on the data will now be described. Each orthogonal function $\boldsymbol{p}_{j}$ in general depends on the independent variables. Let $\boldsymbol{x}_{j}, j=1,2, \ldots, m$ represent $N \mathrm{x} l$ vectors of the $m$ independent variables. Each element of the $\boldsymbol{x}_{j}$ corresponds to one data point. Assign $k$ as a positive integer that serves as a label for a unique set of $m$ non-negative integers $\left\{r_{1}, r_{2}, \ldots, r_{m}\right\}$. For example, if $m=2$, it might be that $k=1$ corresponds to $\{0,0\}, k=2$ corresponds to $\{0,1\}, k=3$ corresponds to $\{1,0\}, k=4$ corresponds to $\{0,2\}, k=5$ corresponds to $\{1,1\}$, and so on. Orthogonal function $p_{k}$ is associated with the $k^{\text {th }}$ set of $m$ non-negative integers, and also with the ordinary polynomial function $w_{k}$ of the $m$ independent variables:

$$
w_{k}=x_{1}^{r_{1}} \otimes x_{2}^{r_{2}} \otimes \ldots \otimes x_{m}^{r_{m}}
$$


where, for example, $\boldsymbol{x}_{1}^{r_{1}}$ denotes an $N$-dimensional vector with each element of the independent variable vector $x_{1}$ raised to the $r_{1}$ power, and $\otimes$ denotes an element-by-element product. Note that each $\boldsymbol{w}_{k}$ is an ordinary polynomial in the independent variables. Define the order of $w_{k}$ as

$$
\varphi(k)=r_{1}+r_{2}+\ldots+r_{m}
$$

The orthogonal functions of $m$ independent variables are defined by the following generating relations:

$$
p_{1}=I
$$

where $I$ represents an $N \times I$ vector of ones and $k=1$ is associated with the set of $m$ zeros, $r_{1}=r_{2}=\ldots=r_{m}=0$.

Each new set $k$ evolves from a previous set $\tilde{k}$, related as follows:

$$
\begin{aligned}
& \tilde{k} \Leftrightarrow\left\{r_{1}, r_{2}, \ldots, r_{\mu-1}, r_{\mu}, r_{\mu+1}, \ldots, r_{m}\right\} \\
& k \Leftrightarrow\left\{r_{1}, r_{2}, \ldots, r_{\mu-1}, r_{\mu}+1, r_{\mu+1}, \ldots, r_{m}\right\}
\end{aligned}
$$

where $\mu$ is an integer. In Eq. (21), the only difference between the integers in set $\widetilde{k}$ and those in set $k$ is that the integer index for independent variable $\mu$ in set $k$ is one more than in set $\tilde{k}$. By assumption, the $\tilde{k}^{\text {th }}$ orthogonal function has already been generated. The orthogonal function $\boldsymbol{p}_{k}$ is then generated by

$$
p_{k}=x_{\mu} \otimes p_{\widetilde{k}}-\sum_{j} \gamma_{j}^{k} p_{j}
$$

with the summation over all $j<k$ such that $\varphi(k)-\varphi(j) \leq 2$. The $\gamma_{j}^{k}$ are constants to be determined. The index $k$ and its associated integer set keep track of the independent variable orders for the $k^{\text {th }}$ orthogonal function. Each new orthogonal function must be orthogonal only to the previously generated orthogonal functions of the same order, one order lower, and two orders lower to be orthogonal to the entire set of generated orthogonal functions. Proof of this was found by Weisfeld ${ }^{9}$. Orthogonality was verified numerically in the software implementation of the technique used in this work.
The scalars $\gamma_{j}^{k}$ are computed for each $j$ by multiplying both sides of Eq. (22) by $\boldsymbol{p}_{j}^{T}$ and invoking the orthogonality of the $\boldsymbol{p}_{j}$ from Eq. (10),

$$
\gamma_{j}^{k}=\frac{\boldsymbol{p}_{j}^{T}\left(\boldsymbol{x}_{\mu} \otimes \boldsymbol{p}_{\bar{k}}\right)}{\boldsymbol{p}_{j}^{T} \boldsymbol{p}_{j}}
$$

The quantities on the right in Eq. (23) are either a measured independent variable vector or a previously generated orthogonal function. After the scalars $\gamma_{j}^{k}$ are determined from Eq. (23) for each $j, p_{k}$ can be computed from Eq. (22).

The process can be repeated to generate orthogonal functions of arbitrary order in the independent variables, subject only to limitations related to the information contained in the data. Speaking loosely, the multivariate orthogonal functions $\boldsymbol{p}_{k}$ are orthogonalized versions of the corresponding ordinary polynomial functions $\boldsymbol{w}_{k}$.

\section{Parameter Estimation}

Once the orthogonal functions to be included in the model of Eq. (1) were generated by Eqs. (20), (22) and (23), then selected by minimizing the PSE from Eq. (14), each retained orthogonal function was decomposed into an expansion of ordinary polynomial functions in the independent variables. This step introduced negligible error, as described below.

From the orthogonal function generating equation (22) and the discussion in the previous section, it can be deduced that for any index $k$, the orthogonal function $\boldsymbol{p}_{k}$ is a linear combination of the $\boldsymbol{w}_{i}$ for all $i \leq k$. In other words, each orthogonal function $p_{k}$ can be expressed as a linear combination of the $\boldsymbol{w}_{i}, i=1,2, \ldots, k$, which are ordinary polynomials corresponding to the integer sets of all previously generated orthogonal functions plus the current $\left(k^{\text {th }}\right)$ one. In equation form,

$$
\boldsymbol{p}_{k}=b_{k 1} \boldsymbol{w}_{1}+b_{k 2} \boldsymbol{w}_{2}+\ldots+b_{k k} \boldsymbol{w}_{k}
$$

where the $b_{k i}, i=1,2, \ldots, k$, are constants to be determined. There is no question as to the model structure given for each $\boldsymbol{p}_{k}$ in Eq. (24), because this 
model structure was guaranteed by the use of Eq. (22) in generating each orthogonal function.

The $b_{k i}, i=1,2, \ldots, k$ in Eq. (24) needed for the decomposition of each orthogonal function were computed using a conventional least squares solution, as given in Eqs. (5) and (6). Since the form and number of terms needed for the ordinary polynomial expansion of each orthogonal function was known, the decomposition of the orthogonal functions into an ordinary polynomial expansion introduced negligible error - on the order of the numerical precision $\left(10^{-12}\right)$ - for each orthogonal function. It would also be possible to find the $b_{k i}$ parameters in Eq. (24) exactly, by bookkeeping and properly combining the $\gamma_{j}^{k}$ values.

When all retained orthogonal functions were decomposed, the expansions like Eq. (24) were substituted into Eq. (1) for each retained orthogonal function, and common terms in the ordinary polynomials were combined using double precision arithmetic to arrive finally at a multivariate model using only ordinary polynomials in the independent variables. Ordinary polynomial terms that contributed less than 0.1 percent of the final model output rootmean-square magnitude were dropped. The computer program that implements the procedure described here can determine up to $8^{\text {th }}$ order models with a maximum of 10 independent variables.

In summary, a model given by Eq. (1) of the dependent variable in terms of orthogonal functions $\boldsymbol{p}_{j}$ was determined using the minimum PSE criterion. Each $\boldsymbol{p}_{j}$ in Eq. (1) was then decomposed into an expansion of ordinary polynomial functions, with the results combined to arrive at a model of the form:

$$
\hat{\boldsymbol{y}}=\hat{c}_{i_{1}} \boldsymbol{w}_{i_{1}}+\hat{c}_{i_{2}} \boldsymbol{w}_{i_{2}}+\ldots+\hat{c}_{i_{n_{p}}} \boldsymbol{w}_{i_{n_{p}}}
$$

with

$$
i_{1}, i_{2}, \ldots, i_{n_{p}} \in\left\{1,2, \ldots, k_{\max }\right\}
$$

where $k_{\max }$ is the total number of orthogonal functions generated and $w_{i_{1}}, w_{i_{2}}, \ldots, w_{i_{n_{p}}}$ are ordinary polynomials in the independent variables. The positive integer values of $i_{1}, i_{2}, \ldots, i_{n_{p}}$ and $n_{p}$ depend on the particular orthogonal functions retained in the model structure determination stage and also on the subsequent decomposition of each retained orthogonal function in terms of ordinary polynomials. The number of ordinary polynomial terms $n_{p}$ may be different than $n$ from the orthogonal function expansion.

The orthogonal function model of Eq. (1) was useful in determining the model structure for the dependent variable using the minimum PSE criterion, by virtue of the properties of orthogonal functions and the resultant decoupling of the associated least squares problem. The subsequent decomposition of the retained orthogonal functions was done to express the results in physically meaningful terms and to allow analytical differentiation for derivatives of the dependent variable with respect to the independent variables.

\section{Experimental Data}

The wind tunnel data used in this work were recently acquired in the National Transonic Facility (NTF) at NASA Langley Research Center. The model was a commercial jet transport, although nothing about the current analytical method is limited to this class of aircraft, which was selected for illustrative purposes. Non-dimensional aerodynamic lift, drag, and pitching moment coefficient values were measured for various angles of attack and Mach numbers within the cruise flight envelope of the vehicle. The independent variables were angle of attack and Mach number. The response variables were non-dimensional aerodynamic coefficients for lift force, drag force, and pitching moment.

The wind tunnel data was collected using two different experiment designs, MDOE and OFAT, over roughly the same range of angle of attack and Mach number. For the MDOE experiment, the independent variables were set according to a $2^{\text {nd }}$ order Box-Wilson design including far-comer points, with additional points from a custom $3^{\text {rd }}$ order design 11 that was based on an orthogonally blocked extension to a Box-Wilson design. For the OFAT experiment, angle of attack settings were increased monotonically with time for various constant Mach number settings.

The MDOE data from the full wind tunnel test were divided into 9 subspaces, shown in Table 1. Within each subspace, independent variables were set according to the normalized values contained in Table 2. Normalized independent variable values can be found by mapping the independent variable values in engineering units for each subspace onto the interval 
$[-1,1]$. The normalization of each independent variable was implemented by

$$
\widetilde{x}=-1+2 \frac{\left(x-x_{\min }\right)}{\left(x_{\max }-x_{\min }\right)}
$$

where $\widetilde{x}$ was the normalized value of the independent variable, and the independent variable range in engineering units was $\left[x_{\min }, x_{\max }\right]$. The inverse transformation was

$$
x=x_{\min }+\frac{(\tilde{x}+1)}{2}\left(x_{\max }-x_{\min }\right)
$$

For example, a normalized value of 0.707 for angle of attack in subspace 2 would correspond to 3.634 degrees in engineering units, since the angle of attack interval for subspace 2 was $[1.5,4]$ deg. Similarly, the normalized Mach number setting -0.707 corresponds to Mach number 0.718 for subspace 2, with Mach number interval $[0.7,0.82]$. All modeling and prediction in this work was done using normalized values of the independent variables.

\section{Results}

The multivariate orthogonal function nonlinear modeling technique was first applied to experimental data from the individual MDOE subspaces in Table 1.

Values for $\sigma_{\max }^{2}$ were found from the repeated runs at the normalized center points of the independent variable ranges, using the method described above. Model structure determination and parameter estimation was done automatically using the orthogonal function modeling technique, without iteration or analyst judgment of any kind. While the orthogonal function modeling software allows manual override by the analyst in the model structure determination stage, this option was not used for any of the results presented here. All data analysis and modeling was done on a $450 \mathrm{MHz}$ computer running Matlab 5.3. The orthogonal modeling technique was implemented as a Matlab $\mathrm{m}$-file $\mathrm{e}^{10}$.

Figure 2 shows the response surface model fit to measured lift coefficient data for the normalized independent variable values listed in Table 2 for subspace 2 of Table 1. The crosses shown in Figure 2 are the measured data used for the modeling. The middle plot in Figure 3 shows the residuals for the lift coefficient model, which are the differences between the measured lift coefficient data and the response surface model values. The solid horizontal lines are the $95 \%$ confidence bounds for the prediction error of the response surface model, computed as two times the square root of the PSE from Eq. (14). The prediction error bound calculation therefore used modeling data to estimate the range for the residuals in prediction cases.

The circles in Figure 3 are prediction errors computed for 5 data points not used for the modeling. This data was collected at the randomly selected normalized independent variable settings given in Table 3. For these points, the identified response surface model was used to predict the measured output. Figure 3 shows that the $95 \%$ confidence bound for the prediction error computed from PSE was a conservative measure of actual prediction error, indicated by the circles. The prediction errors were roughly the same magnitude as the residuals for the modeling data (or inference data), marked by $x$ 's. This indicates that the functional dependence was captured well by the identified response surface model.

The other plots in Figure 3 show similar information for the drag force and pitching moment coefficients. Table 4 contains detailed information about the modeling and prediction for this case. The first two rows of Table 4 show the mean values of the coefficients and estimates of the standard deviation of the output noise from repeated center point runs. This gives a measure of the output noise level and the how the noise magnitude compares to the mean measured output. Residual magnitudes close to the noise level is the best that any model can be expected to do. The third row shows the prediction error estimated from the modeling data, and the fourth row shows the root mean square of the modeling error using the independent variable settings in Table 2 for subspace 2 of Table 1. The fifth row of Table 4 shows the root mean square of the actual prediction error using the identified response surface model to predict the 5 points from Table 3 . The prediction error estimate based on the modeling data was conservative (i.e., too large) by approximately a factor of 4 for this data. The actual prediction performance was nearly the best that it could be, considering the values for the estimated output noise $\sigma_{o}$, compared to the actual prediction errors. The actual prediction errors were less than 1 percent of the mean values for all coefficients, indicating an excellent prediction capability for the identified response surface models. Finally, the last three rows of Table 4 indicate the number of orthogonal function terms in each identified response surface model, the number of 
ordinary polynomial terms in each model after decomposing the orthogonal functions, and the maximum order of the ordinary polynomial terms in the model.

Similar analysis was done for the other subspaces defined in Table 1. The results showed that prediction errors estimated from the modeling data were always conservative, but by varying amounts. Also, the model fit errors and prediction errors were larger for the higher Mach number cases (subspaces 4-9), because of larger output noise values, estimated by $\sigma_{o}$.

Response surface modeling was repeated for subspace 2 using only the $2^{\text {nd }}$ order Box-Wilson and far-corner points from the original experiment design in Table 2. Results plotted in Figure 4 show that model fit errors and prediction errors were generally larger, but the prediction error estimates from modeling data were still conservative. The increased number of prediction points in Figure 4 resulted from treating the unused $3^{\text {rd }}$ order points in Table 2 as additional prediction cases.

Figures 5 and 6 show results from response surface modeling based on the $2^{\text {nd }}$ order Box-Wilson design and the $3^{\text {rd }}$ order augmented design, excluding the far-corner points in Table 2. In these cases, some prediction errors were outside prediction error bound estimates based on the modeling data. All of these outliers were in fact far-comer points, which were included as prediction points for these cases. In this context, the far-corner points were extrapolations for the identified models, with prediction errors higher than the estimates based on the modeling data. Including the far-corner points in the modeling decreased the prediction errors for both $2^{\text {nd }}$ and $3^{\text {rd }}$ order designs individually. In addition, use of the $3^{\text {rd }}$ order experiment design with far-corner points decreased the prediction error compared to using the $2^{\text {nd }}$ order Box-Wilson experiment design with farcorner points. When the far-corner points were not included, models identified from the $3^{\text {rd }}$ order experiment design had higher prediction errors than models identified from the $2^{\text {nd }}$ order Box-Wilson design. All of this behavior was consistent for data from the other subspaces in Table 1.

Data from a larger independent variable subspace was also analyzed. Specifically, all the test points in Table 2 for subspaces 1, 2, and 3 in Table I were combined into a single MDOE data set. Figure 7 shows the modeling and prediction results in the same format as before, for all three longitudinal aerodynamic coefficients. Table 5 contains the modeling and prediction results. The prediction points were chosen randomly in the independent variable space corresponding to subspaces 1,2 , and 3 in Table 1 .

Response surface modeling was then done for the same independent variable space corresponding to subspaces 1, 2, and 3, but using data from an OFAT experiment design. For this experiment, several sweeps through the angle of attack range were made for 4 different Mach numbers. Table 6 contains the modeling and prediction results, and Figure 8 shows the plots for this case. The same number of data points (90) were used for the OFAT data set as for the MDOE data set. The response surface model identified from the OFAT data was applied to the same prediction data points used with the MDOE data. Comparing the information in Tables 5 and 6, the models identified from the MDOE and OFAT data were of comparable complexity, and the model fit errors were nearly the same, except for the drag force coefficient, which was fit better using the MDOE data. Prediction errors were lower for lift and drag force coefficient models identified from the MDOE data. The prediction error for the drag coefficient was significantly lower (by roughly a factor of 3 ) using MDOE data, compared to using OFAT data.

Figures 7 and 8 show that the orthogonal function modeling technique did well with either MDOE or OFAT data, in terms of fitting the model data accurately, estimating prediction error from model data, and actual prediction, except for the degraded prediction with the drag coefficient model using OFAT data, noted earlier. All prediction errors and model errors in Tables 5 and 6 were less than 4 percent of the mean values for all aerodynamic coefficients.

Finally, Table 7 contains response surface modeling results for subspaces 1,2 , and 3, using only $2^{\text {nd }}$ order Box-Wilson points, including far corner points. These data represent a subset of the MDOE data used for Table 5 and Figure 7. Using the MDOE subset of $2^{\text {nd }}$ order points with far comers (Table 7), the prediction error for all output response surface models was increased compared to the response surface models identified from all MDOE data (Table 5). This behavior was similar to what was seen in Figures 3 and 4 for MDOE subspace 2 data. Compared to the models identified from OFAT data, prediction errors using the models identified from the MDOE $2^{\text {nd }}$ order subset data were lower for $C D$ and $C L$, but higher for CM. The number of data points used to identify the models in Table 7 was 60 , compared to 90 for Tables 5 and 6. 


\section{Concluding Remarks}

A nonlinear modeling technique based on the use of multivariate orthogonal modeling functions generated from the measured data was used to characterize response surfaces for wind tunnel data. A minimum prediction error criterion was used to select which multivariate orthogonal functions should be included in the model. When used with orthogonal modeling functions, this prediction error metric always has a single global minimum. The global minimum was used to automatically identify which orthogonal modeling functions should be included in the response surface model. The orthogonal functions were generated in a manner that allows them to be decomposed without ambiguity into an expansion of ordinary multivariate polynomials. This allowed the final form of the model to be converted to a multivariate ordinary polynomial expansion in the independent variables, which can provide physical insight into the identified functional dependencies.

The multivariate orthogonal function modeling technique was applied to various subsets of wind tunnel data for a commercial jet transport model, and was shown to be capable of accurately identifying response surface models for experimental data with nonlinear effects. No iteration or analyst judgment was required. The identified response surface models fit the modeling data well in all cases. The model fit errors and prediction errors were smaller when the range of independent variables in the data was smaller (i.e., the inference space was smaller). Prediction capability of all identified models was tested. Prediction errors were estimated somewhat conservatively (i.e., overestimated) using $95 \%$ confidence bounds based on the prediction error metric used to identify the response surface models. Actual prediction errors were within the computed $95 \%$ confidence bounds, except for prediction points that were extrapolations outside the independent variable space used to identify the model.

Response surface models were identified for a relatively large inference space using experimental data collected with both a Modern Design Of Experiments (MDOE) approach, and a One Factor At a Time (OFAT) approach. The multivariate orthogonal function modeling technique worked well with both data sets, achieving less than 4 percent error in both modeling and prediction for all cases. Using the same number of data points, drag coefficient model fit and predictions were significantly degraded using OFAT data, compared to using MDOE data. Models identified from MDOE data with fewer test points than
OFAT data achieved lower prediction errors for lift and drag response predictions, but not for pitching moment.

\section{References}

1. DeLoach, R. "Applications of Modern Experiment Design to Wind Tunnel Testing at NASA Langley Research Center," AIAA 2000-2639, 36 th AIAA Aerospace Sciences Meeting and Exhibit, Reno, NV, January 1998.

2. Fisher, R.A., The Design of Experiments, $8^{\text {th }}$ Ed., Oliver and Boyd, Edinburgh, UK, 1966.

3. Box, G.E.P., Hunter, W.G., and Hunter, J.S., Statistics for Experimenters - An Introduction to Design, Data Analysis, and Model Building, John Wiley \& Sons, Inc., New York, NY, 1978, Chapter 14.

4. Draper, D.S. and Smith, R.L., Applied Regression Analysis, $3^{\text {rd }}$ Ed., John Wiley \& Sons, New York, NY, 1987.

5. Klein, V., Batterson, J.G., and Murphy, P.C., "Determination of Airplane Model Structure from Flight Data by Using Modified Stepwise Regression," NASA TP-1916, 1981.

6. Lo, C.F., Zhao, J.L., and DeLoach, R., "Application of Neural Networks to Wind Tunnel Data Response Surface Methods," AIAA 2000-2639, $21^{\text {St }}$ AIAA Aerodynamic Measurement Technology and Ground Testing Conference, Denver, CO, June 2000.

7. Morelli, E.A., "Global Nonlinear Aerodynamic Modeling using Multivariate Orthogonal Functions," Journal of Aircraft, Vol. 32, No. 2, March-April 1995, pp. 270-77.

8. Barron, A.R., "Predicted Squared Error : A Criterion for Automatic Model Selection," Self-Organizing Methods in Modeling, Farlow, S.J., Ed., Marcel Dekker, Inc., New York, NY, 1984, pp. 87-104.

9. Weisfeld, M., "Orthogonal Polynomials in Several Variables," Numerische Mathematik, Vol. 1, 1959, pp. 38-40.

10. Using MATLAB, Version 5, The MathWorks, Inc., Natick, MA, 1997.

11. Line, W. B. "Third-Order Rotatable Designs in Blocks," DOES Institute, Winston-Salem, NC, 1998. 
Table 1 Inference Subspace Data Ranges

\begin{tabular}{ccccc}
\hline \hline $\begin{array}{l}\text { Inference } \\
\text { Subspace }\end{array}$ & \multicolumn{2}{c}{$\begin{array}{c}\text { Angle of Attack } \\
\text { (deg) }\end{array}$} & \multicolumn{2}{c}{ Mach Number } \\
\hline & $\min$ & $\max$ & $\min$ & $\max$ \\
\hline 1 & -2.5 & 1.5 & 0.7 & 0.82 \\
\hline 2 & 1.5 & 4 & 0.7 & 0.82 \\
\hline 3 & 4 & 6 & 0.7 & 0.82 \\
\hline 4 & -2.5 & 1 & 0.82 & 0.865 \\
\hline 5 & 1 & 3.5 & 0.82 & 0.865 \\
\hline 6 & 3.5 & 6 & 0.82 & 0.865 \\
\hline 7 & -2.5 & 1 & 0.865 & 0.92 \\
\hline 8 & 1 & 3 & 0.865 & 0.92 \\
\hline 9 & 3 & 6 & 0.865 & 0.92 \\
\hline \hline
\end{tabular}

Table 2 Normalized Independent Variable Values

\begin{tabular}{|c|c|c|c|}
\hline & $\begin{array}{c}\text { Point } \\
\text { Number }\end{array}$ & $\begin{array}{l}\text { Angle of } \\
\text { Attack }\end{array}$ & $\begin{array}{l}\text { Mach } \\
\text { Number }\end{array}$ \\
\hline \multirow{16}{*}{ 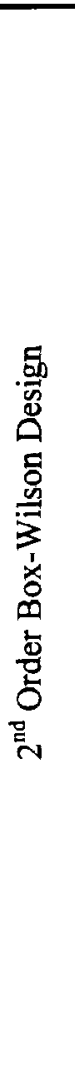 } & 1 & -0.707 & -0.707 \\
\hline & 2 & 0.707 & -0.707 \\
\hline & 3 & -0.707 & 0.707 \\
\hline & 4 & 0.707 & 0.707 \\
\hline & 5 & 0 & 0 \\
\hline & 6 & 0 & 0 \\
\hline & 7 & 0 & 0 \\
\hline & 8 & 0 & 0 \\
\hline & 9 & 0 & -1 \\
\hline & 10 & 0 & 1 \\
\hline & 11 & -1 & 0 \\
\hline & 12 & 1 & 0 \\
\hline & 13 & 0 & 0 \\
\hline & 14 & 0 & 0 \\
\hline & 15 & 0 & 0 \\
\hline & 16 & 0 & 0 \\
\hline \multirow{4}{*}{ 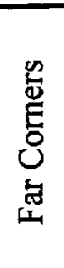 } & 17 & -1 & -1 \\
\hline & 18 & 1 & -1 \\
\hline & 19 & -1 & l \\
\hline & 20 & 1 & 1 \\
\hline \multirow{10}{*}{ 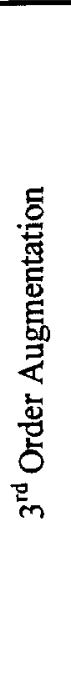 } & 21 & -0.559 & -0.559 \\
\hline & 22 & 0.559 & -0.559 \\
\hline & 23 & -0.559 & 0.559 \\
\hline & 24 & 0.559 & 0.559 \\
\hline & 25 & 0 & -0.791 \\
\hline & 26 & 0 & 0.791 \\
\hline & 27 & -0.791 & 0 \\
\hline & 28 & 0.791 & 0 \\
\hline & 29 & 0 & 0 \\
\hline & 30 & 0 & 0 \\
\hline
\end{tabular}


Table 3 Inference Subspace 2

Prediction Data Points

\begin{tabular}{|c|c|c|c|c|}
\hline & \multicolumn{2}{|c|}{$\alpha(\mathrm{deg})$} & \multicolumn{2}{|c|}{$M$} \\
\hline $\begin{array}{l}\text { Point } \\
\text { Number }\end{array}$ & $\begin{array}{l}\text { Eng. } \\
\text { Units }\end{array}$ & $\begin{array}{l}\text { Norm. } \\
\text { Units }\end{array}$ & $\begin{array}{l}\text { Eng. } \\
\text { Units }\end{array}$ & $\begin{array}{l}\text { Norm. } \\
\text { Units }\end{array}$ \\
\hline 1 & 1.5168 & -0.9865 & 0.8080 & 0.8000 \\
\hline 2 & 3.2098 & 0.3679 & 0.7120 & -0.8000 \\
\hline 3 & 2.2758 & -0.3794 & 0.7840 & 0.4000 \\
\hline 4 & 1.8696 & -0.7043 & 0.7600 & 0.0000 \\
\hline 5 & 1.8917 & -0.6866 & 0.7360 & -0.4000 \\
\hline
\end{tabular}

Table 4 MDOE Data, Inference Subspace 2 Modeling and Prediction Results

\begin{tabular}{c|ccc}
\hline & $\mathrm{CD}$ & $\mathrm{CL}$ & $\mathrm{CM}$ \\
\hline Mean Value & 0.0281 & 0.4817 & -0.0678 \\
\hline $\begin{array}{c}\sigma_{o} \\
\left(\times 10^{4}\right)\end{array}$ & 0.414 & 4.878 & 1.649 \\
\hline $\begin{array}{c}\sqrt{P S E} \\
\left(\times 10^{4}\right)\end{array}$ & 1.947 & 20.000 & 7.362 \\
\hline $\begin{array}{c}\text { Model Error } \\
\sqrt{\hat{J} / N}\left(\times 10^{4}\right)\end{array}$ & 0.817 & 6.066 & 3.727 \\
\hline $\begin{array}{c}\text { Prediction Error } \\
\left(\mathrm{x} 10^{4}\right)\end{array}$ & 0.477 & 5.140 & 1.593 \\
\hline $\begin{array}{c}\text { Orthogonal } \\
\text { Function Terms }\end{array}$ & 11 & 9 & 9 \\
\hline $\begin{array}{c}\text { Ordinary } \\
\text { Polynomial Terms }\end{array}$ & 11 & 9 & 9 \\
\hline $\begin{array}{c}\text { Maximum Order } \\
\text { of Ordinary }\end{array}$ & 4 & 3 & 3 \\
\hline Polynomial Terms & & & \\
\hline
\end{tabular}

Table 5 MDOE Data, Subspaces 1, 2, and 3 Modeling and Prediction Results

\begin{tabular}{c|ccc}
\hline & $\mathrm{CD}$ & $\mathrm{CL}$ & $\mathrm{CM}$ \\
\hline Mean Value & 0.032 & 0.447 & -0.067 \\
\hline $\begin{array}{c}\sigma_{o} \\
\left(\times 10^{4}\right)\end{array}$ & 0.721 & 9.063 & 1.980 \\
\hline $\begin{array}{c}\sqrt{P S E} \\
\left(\times 10^{4}\right)\end{array}$ & 3.274 & 47.925 & 19.936 \\
\hline $\begin{array}{c}\text { Model Error } \\
\sqrt{\hat{J} / N}\left(\times 10^{4}\right)\end{array}$ & 2.527 & 41.274 & 19.043 \\
\hline $\begin{array}{c}\text { Prediction Error } \\
\left(\times 10^{4}\right)\end{array}$ & 2.604 & 46.550 & 20.221 \\
\hline $\begin{array}{c}\text { Orthogonal } \\
\text { Function Terms }\end{array}$ & 15 & 13 & 16 \\
\hline $\begin{array}{c}\text { Ordinary } \\
\text { Polynomial Terms }\end{array}$ & 20 & 20 & 20 \\
\hline $\begin{array}{c}\text { Maximum Order } \\
\text { of Ordinary }\end{array}$ & 6 & 6 & 6 \\
Polynomial Terms & & & \\
\hline \hline
\end{tabular}

Table 6 OFAT Data, Subspaces 1, 2, and 3 Modeling and Prediction Results

\begin{tabular}{c|ccc}
\hline & $\mathrm{CD}$ & $\mathrm{CL}$ & $\mathrm{CM}$ \\
\hline Mean Value & 0.030 & 0.441 & -0.069 \\
\hline $\begin{array}{c}\sigma_{o} \\
\left(\times 10^{4}\right)\end{array}$ & 0.721 & 9.063 & 1.980 \\
\hline $\begin{array}{c}\sqrt{P S E} \\
\left(\times 10^{4}\right)\end{array}$ & 4.059 & 49.362 & 21.202 \\
\hline $\begin{array}{c}\text { Model Error } \\
\sqrt{\hat{J} / N}\left(\times 10^{4}\right)\end{array}$ & 3.494 & 41.605 & 20.242 \\
\hline $\begin{array}{c}\text { Prediction Error } \\
\left(\times 10^{4}\right)\end{array}$ & 7.945 & 52.524 & 19.942 \\
\hline $\begin{array}{c}\text { Orthogonal } \\
\text { Function Terms }\end{array}$ & 15 & 15 & 17 \\
\hline $\begin{array}{c}\text { Ordinary } \\
\text { Polynomial Terms }\end{array}$ & 17 & 20 & 19 \\
\hline $\begin{array}{c}\text { Maximum Order } \\
\text { of Ordinary }\end{array}$ & 5 & 6 & 6 \\
Polynomial Terms & & & \\
\hline \hline
\end{tabular}


Table 7 MDOE Data, Subspaces 1, 2, and 3 $2^{\text {nd }}$ Order Box-Wilson with Far Corners Modeling and Prediction Results

\begin{tabular}{c|ccc}
\hline \hline & $\mathrm{CD}$ & $\mathrm{CL}$ & $\mathrm{CM}$ \\
\hline Mean Value & 0.033 & 0.447 & -0.067 \\
\hline $\begin{array}{c}\sigma_{o} \\
\left(\times 10^{4}\right)\end{array}$ & 0.703 & 9.836 & 1.483 \\
\hline $\begin{array}{c}\sqrt{P S E} \\
\left(\mathrm{x} 10^{4}\right)\end{array}$ & 3.570 & 48.539 & 16.320 \\
\hline $\begin{array}{c}\text { Model Error } \\
\sqrt{\hat{J} / N}\left(\mathrm{x} 10^{4}\right)\end{array}$ & 2.667 & 37.263 & 15.094 \\
\hline $\begin{array}{c}\text { Prediction Error } \\
\left(\mathrm{x} 10^{4}\right)\end{array}$ & 3.116 & 49.331 & 22.664 \\
\hline $\begin{array}{c}\text { Orthogonal } \\
\text { Function Terms }\end{array}$ & 14 & 12 & 21 \\
\hline $\begin{array}{c}\text { Ordinary } \\
\text { Polynomial Terms }\end{array}$ & 19 & 20 & 22 \\
\hline $\begin{array}{c}\text { Maximum Order } \\
\text { of Ordinary }\end{array}$ & 6 & 6 & 6 \\
Polynomial Terms & & & \\
\hline
\end{tabular}




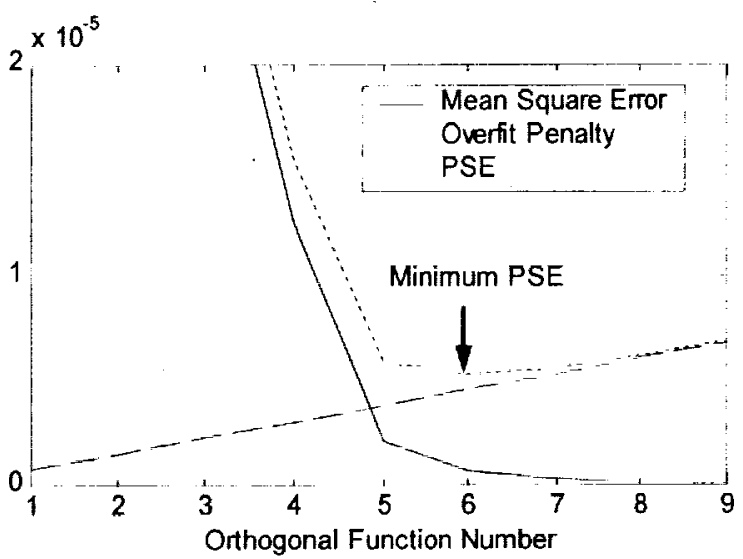

Figure 1 Predicted Squared Error (PSE) Components

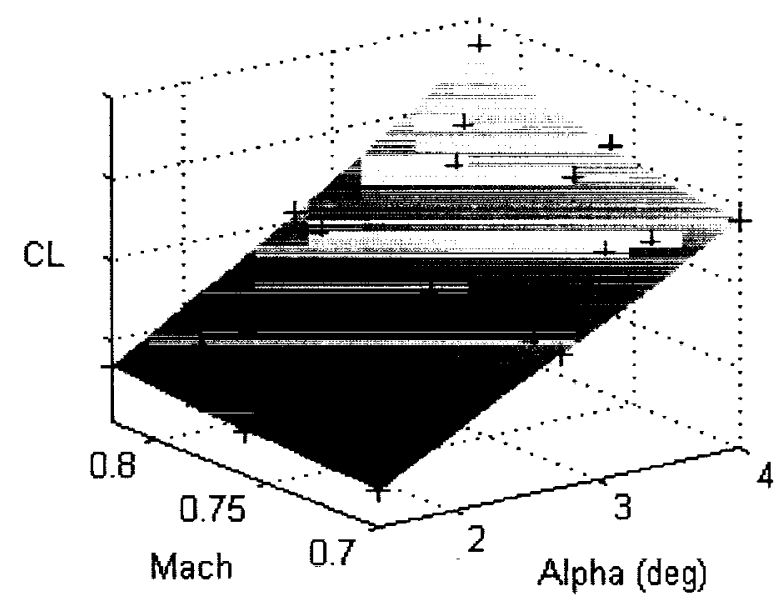

Figure 2 Response Surface Model Fit Lift Coefficient, Subspace 2
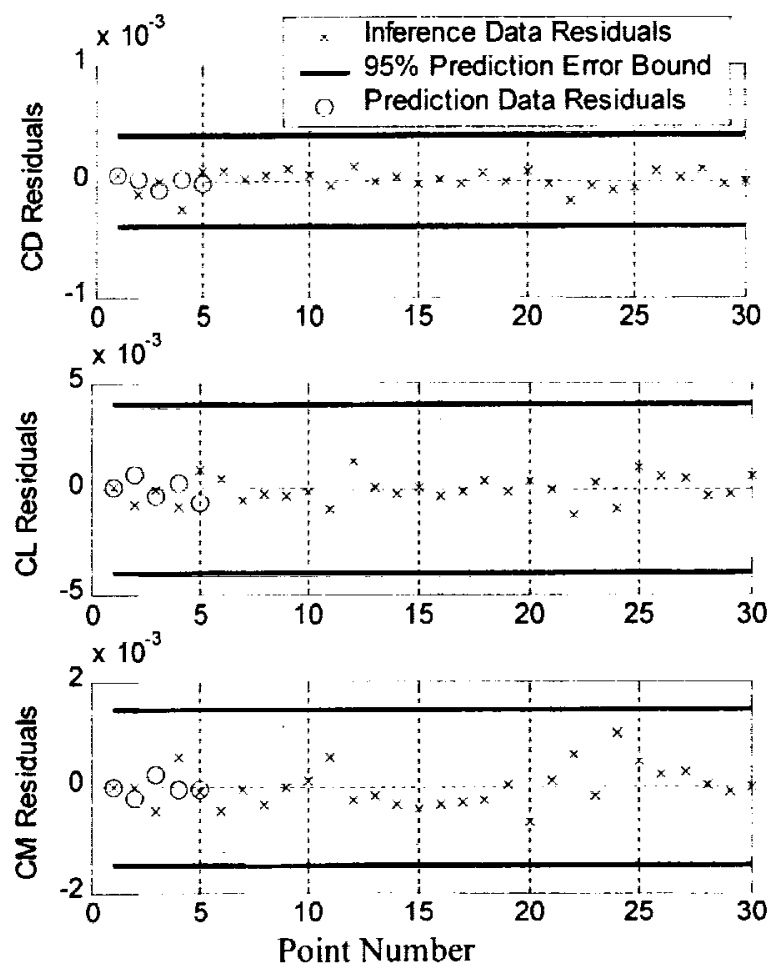

Figure $33^{\text {rd }}$ Order Data with Far Corners Subspace 2
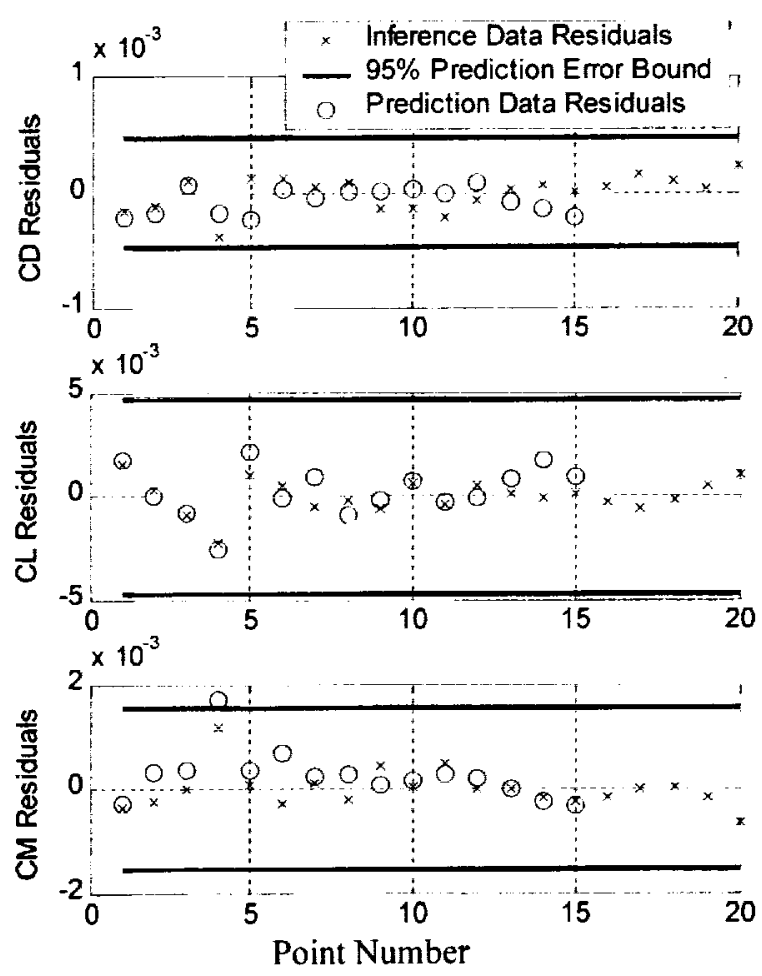

Figure $42^{\text {nd }}$ Order Box-Wilson Data with Far Corners Subspace 2 

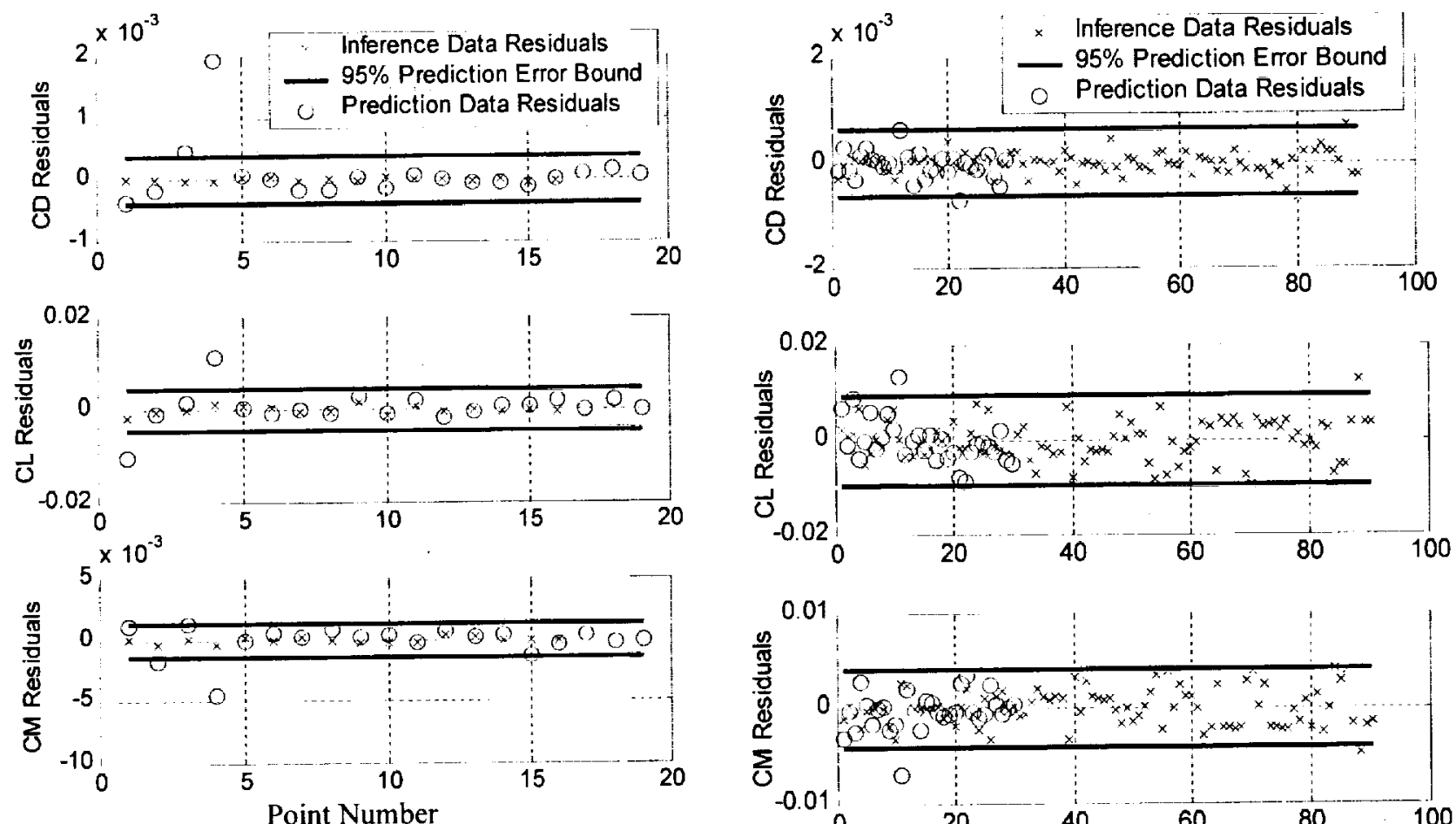

Figure $52^{\text {nd }}$ Order Box-Wilson Data, no Far Corners Subspace 2

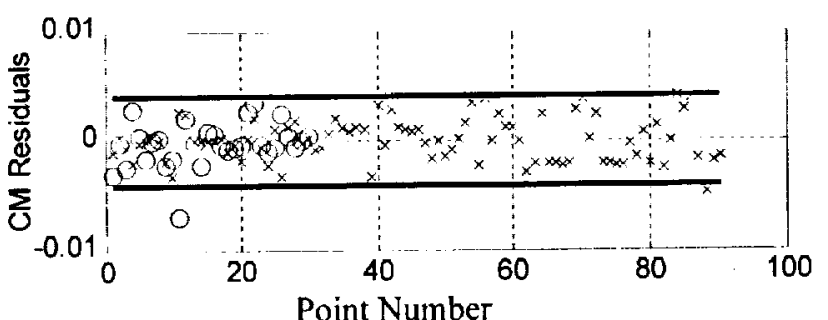

Figure 7 MDOE Data, Subspaces 1, 2, and 3
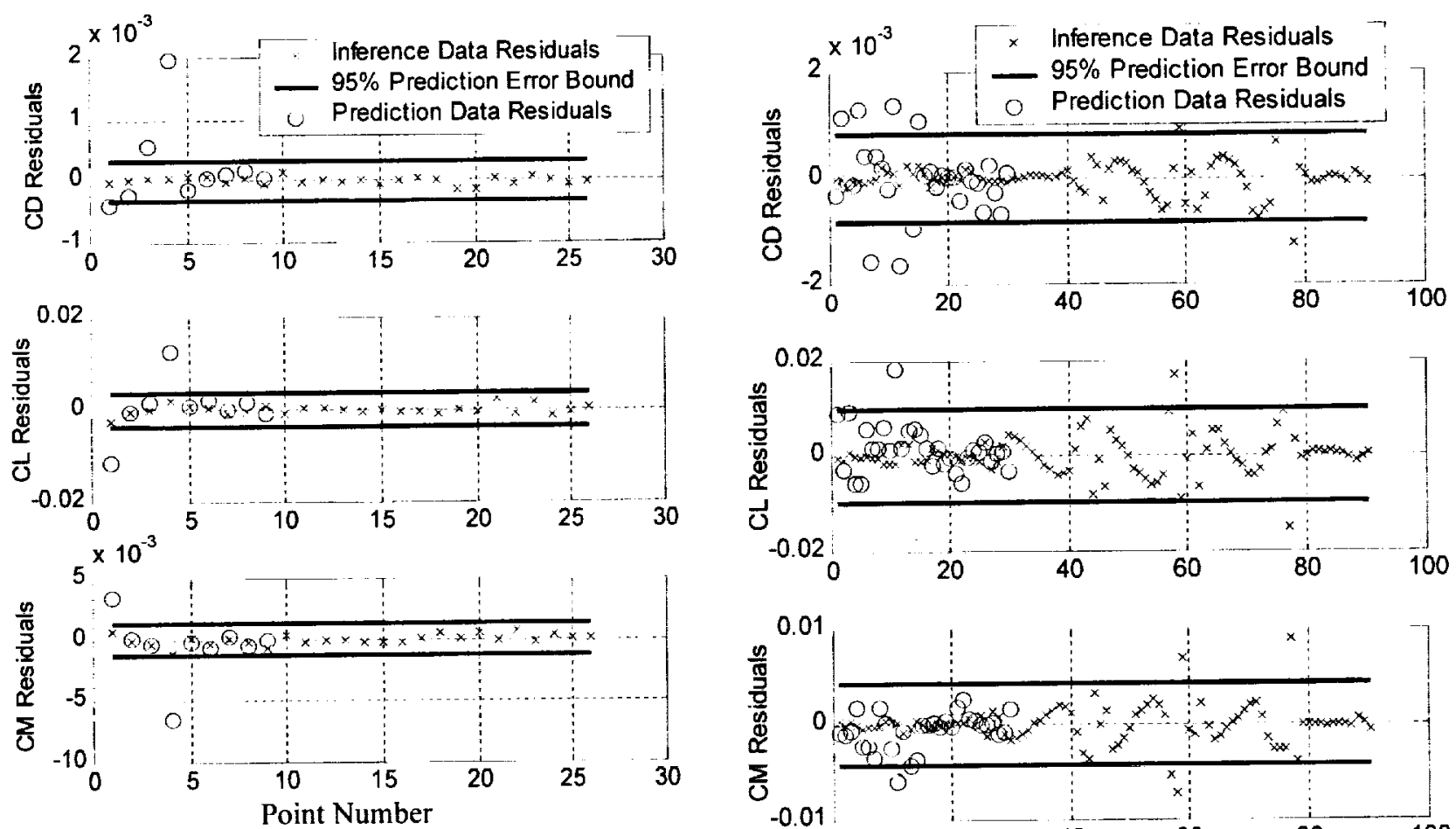

Figure $63^{\text {rd }}$ Order Data, no Far Comers Subspace 2

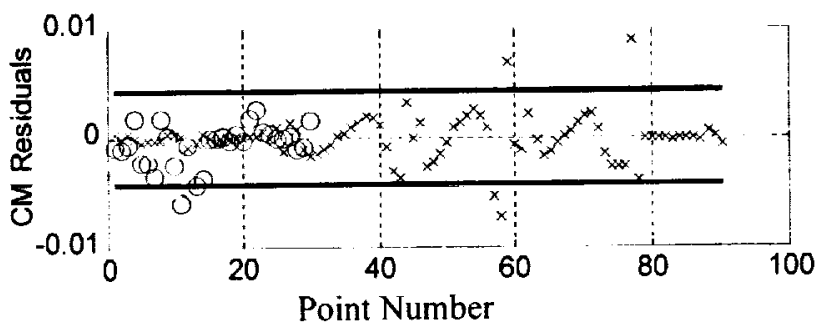

Figure 8 OFAT Data, Subspaces 1, 2, and 3 